\title{
PROBLEMAS DE ADAPTACIÓN FSIOLÓGICA EN PERSONAS CON ANGINA
}

Allyne Nóbrega Fortes*, Viviane Martins da Silva** Marcos Venícios de 0 liveira Lopes***

*Alumna del curso de Master en enfermería de la U niversidad Federal de Ceará

**Licenciada en enfermería, alumna del curso de doctorado en enfermería de la U niversidad Federal de Ceará

***Doctor en Enfermería, Profesor Adjunto del Departamento de Enfermería de la Universidad Federal de Ceará, Fortaleza - Ceará/Brasil.

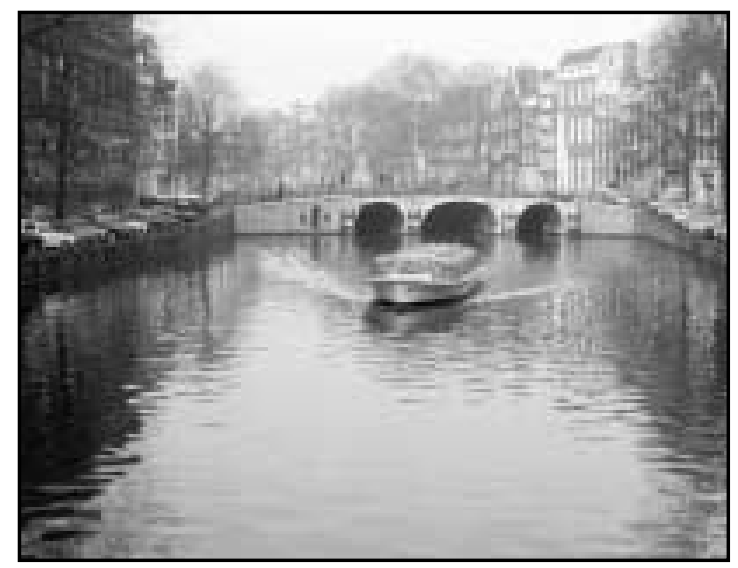

PHYSIOLOGIC ADAPTATION PROBLEMS IN PEOPLE WITH ANGINA

\section{ABSTRACT}

$I^{2}$ $\mathrm{n}$ this article the common physiologic adaptation problems presented by patients with unstable angina were identified, having as a base Callista Roy's Adaptation Model. A descriptive study was carried out in a specialized hospital for cardiac diseases. The sample for the study comprised a total of 20 patients. For characterization and discussion, we used the definitions presented by Roy for each physiologic adaptation problem identified. Regarding common physiologic adaptation problems, the following were identified: Activity intolerance, Constipation, Impairment of a primary sense: vision and audition, Nutrition in excess to body requirements, Sleep deprivation, Potential sleep pattern disturbance, Hyponatremia, Disuse syndrome, Restricted mobility, Anorexia, Memory deficit, Potential ineffective coping with allergic reaction, Stress, Oedema, Hypokalemia, Infection, Instability of Behavior and mood, and Delayed wound healing.

Key words: Unstable Angina, Adaptation, Nursing Theory.

\section{RESUMEN}

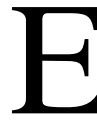

n este artículo fueron identificados los problemas comunes de adaptación fisiológica presentados por pacientes con angina inestable teniendo como base el Modelo de la Adaptación de Callista Roy. Estudio descriptivo desarrollado en un hospital de servicio especializado en enfermedades cardíacas. La muestra para el estudio fue de 20 pacientes. Para caracterización y discusión, utilizamos las definiciones presentadas por Roy para cada problema de adaptación fisiológica identificado. Con relación a los problemas comunes de adaptación fisiológica fueron identificados: Intolerancia a la actividad, Estreñimiento, Deficiencia del sentido primario: visión y audición, Nutrición mayor que las necesidades requeridas, Deprivación de sueño, Riesgo de disturbio del patrón de sueño, Hiponatremia, Síndrome del desuso, Movilidad restrita, Anorexia, Déficit de memoria, Riesgo de enfrentamiento ineficaz con reacción alérgica, Estrés, Edema, Hipocalemia, Infección, Instabilidad de comportamiento y humor, y Cicatrización comprometida.

Palabras-clave: Angina inestable, Adaptación, Teoría de Enfermería. 


\section{INTRODUCCIÓN}

Actualmente los disturbios cardíacos isquémicos vienen creciendo numéricamente a cada año. Un ejemplo de eso es que, en Estados Unidos de América, la estimativa de casos de angina varia de 6 a 16 millones, considerándose la incidencia anual de por lo menos más 150.000 nuevos casos. Tratándose de Brasil existen, por lo menos, 900.000 brasileños con angina del pecho y cerca de 18.000 nuevos casos de la enfermedad ocurriendo a cada año, teniendo en vista un total de 30 casos de angina para cada caso de infarto agudo hospitalizado en un año (Sociedade Brasileira de Cardiologia, 2004).

La angina es un síndrome clínico que se caracteriza por dolor o incomodidad en regiones como: tórax, epigastrio, mandíbula, hombro, dorso o miembros superiores, siendo típicamente impulsada o agravada por actividad física o estrés emocional y atenuada con uso de nitroglicerina y derivados. Ocurre debido a la disminución del flujo coronariano provocado por una constricción de las arterias coronarias. Se sigue por un suministro insuficiente de oxígeno al organismo debido a la demanda aumentada por parte del miocardio que se encuentra isquémico (Robbins, Cotran \& Kumar, 1996).

Además de evaluar el tipo de dolor anginoso por el cual el paciente está sufriendo, es de igual importe que el enfermero evalúe el estímulo que está ocasionando tal cuadro, la comprensión del tratamiento por el paciente y su familia, bien como el proceso de adaptación fisiológica y psicosocial de este individuo al plan de tratamiento actual y a la propia patología.

Para comprender la importancia de la evaluación del nivel de adaptación por el paciente anginoso a esta condición y al posterior tratamiento, escogimos como base teórica de este estudio el modelo de adaptación de Roy, que discute el proceso de adaptación del ser humano a las condiciones impuestas por el entorno (Roy \& Andrews, 1999).

Este modelo aborda inicialmente la noción de estímulos internos (inherentes al ego del individuo) o externos, que pueden ser definidos como un incentivo del ambiente. Ambos pueden ser subdivididos en: focales, contextuales o residuales. Los focales son aquéllos en que el individuo es confrontado prontamente. Los contextuales aparecen en la ocasión y cooperan directamente para el efecto del estímulo focal. Finalmente, los residuales son estímulos personales o ambientales que tienen sus efectos obscuros, mismo existiendo en la situación (Roy \& Andrews, 1999).

El proceso de Enfermería es utilizado en el momento de la actuación del enfermero y de acuerdo con Roy, es dividido en las siguientes fases: evaluación de comportamientos; evaluación de los estímulos; diagnóstico de enfermería; establecimiento de metas, intervenciones y evaluación de las intervenciones desarrolladas (Roy \& Andrews, 1999).

La etapa diagnóstica propuesta por Roy incluye tres formas diferentes de llegarse a un diagnóstico de enfermería: 1) Describiéndose el comportamiento y el estímulo focal identificados; 2) Utilizando la taxonomía de diagnósticos de la NANDA (2005); 3) Utilizando una tipología de problemas comunes de adaptación creada por la propia autora. Visando una mayor aproximación con la visión de la teórica, en este estudio fueron trabajados los problemas comunes de adaptación presentados por pacientes con angina inestable teniendo como base el Modelo de la Adaptación de Callista Roy. El estudio incluyó solamente el Modo de Adaptación Fisiológico

\section{MATERIAL Y MÉTODOS}

Se trata de un estudio descriptivo desarrollado en un hospital especializado en enfermedades cardíacas, vinculado a la red pública de salud de la ciudad de Fortaleza - Ceará - Brasil. La colecta de datos fue realizada en las unidades de internación cardiológica del referido hospital, mediante término de consentimiento aprobado por el departamento de educación continuada del hospital. Esta institución ofrece servicio a pacientes con patologías torácicas, sean respiratorias o cardíacas.

La muestra para el estudio fue definida en un total de 20 pacientes. Para inclusión en la muestra, fueron considerados los siguientes criterios: aceptar voluntariamente la participación en la investigación, de acuerdo con el término de consentimiento; presentar diagnóstico médico de angina inestable, internado en la unidad de internación 
cardiológica del hospital en cuestión; estar en condiciones físicas y emocionales de contestar a las preguntas y de ser sometido al examen físico; tener edad entre 18 y 65 años.

La recogida de datos fue realizada por medio de un formulario aplicado en conjunto con un examen físico en cada paciente estudiado. El formulario fue elaborado basado en un material desarrollado para la aplicación de los Modelos Conceptuales del Proceso de Enfermería. Este guión es compuesto de preguntas específicas dirigidas al Modelo de la Adaptación de Roy, fundamentado en instrumento propuesto por Christensen \& Kenney (1995). Los datos están descritos de forma conceptual y, al lado de cada problema de adaptación, es presentada la frecuencia de su ocurrencia entre paréntesis. Para caracterización y discusión, utilizamos las definiciones presentadas por Roy para cada problema de adaptación fisiológica identificado. Para cada problema de adaptación es discutido solamente el estímulo focal.

El proyecto fue aprobado por el Comité de Ética en Investigación del Hospital. Se solicitó la firma del término de consentimiento por cada participante, autorizando la obtención y la utilización de sus informaciones. Los participantes de la investigación fueron debidamente aclarados en cuanto a la finalidad del estudio, en cuanto a la importancia de su participación en el mismo, y en cuanto a la seguridad de su anonimato.

\section{RESULTADOS Y DISCUSIÓN}

La muestra incluyó 12 hombres y 8 mujeres con edad variando de 41 a 89 años de edad, 14 eran casados y los demás solteros. Del total, 14 eran jubilados y 13 tenían a lo más 5 años de estudios. Más de la mitad (11) tenían antecedentes familiares de enfermedad cardíaca.

La mayoría de los casos de portadores de angina inestable, a ejemplo de las enfermedades cardiovasculares en general, es del sexo masculino. La distribución por edad muestra un grupo más de edad lo que confirma la tendencia de que las coronariopatías, entre ellas la angina inestable, se vuelven más presentes en personas más de edad. La presencia del compañero puede servir como apoyo durante el proceso de enfrentamiento de la enfermedad, de internamiento, en el incentivo en el cambio del estilo de vida no saludable, en el control de los factores de riesgo, en el compañerismo, en el alivio del estrés. El grande número de jubilados es explicable por cuenta de la edad avanzada de los mismos. Esta jubilación significó para muchos de éstos el inicio de una vida sedentaria. Estos jubilados normalmente no practicaban actividad y ni poseían fuente de ocio, lo que deteriora no solo el físico como también el psíquico de estas personas.

Fueron identificados siete factores de riesgo para enfermedades cardíacas que incluyen: hipertensión (9), tabaquismo (5), hipercolesterolemia (4), menopausia (4), accidente vascular cerebral (2), diabetes mellitus (1) y etilismo (1). Entre los factores de riesgo se destaca la hipertensión arterial que afecta diversos componentes del modo de adaptación fisiológica, y su control es fundamental para la efectivación de las respuestas de adaptación. Es importante resaltar que el tabaquismo aún está muy presente en el grupo, presentado por 5 personas de la muestra lo que demuestra que algunos pacientes tienen la dificultad de modificar antiguos hábitos de vida que perjudican su condición de salud. Mientras tanto, factores como etilismo y diabetes están en un margen pequeño de esta muestra.

Con relación a los problemas comunes de adaptación fisiológica identificados, lo más frecuente fue la Intolerancia a la actividad (11) que es definido por Roy como siendo el Estado en el cual el individuo tiene energía fisiológica y psicológica insuficiente para soportar o completar las actividades diarias requeridas o anheladas (Roy \& Andrews, 1999). Registramos relatos de debilidad e indisposición para hacer ejercicios o mismo actividades diarias, incomodidad y disnea a los esfuerzos. El estímulo focal identificado para este problema fue la baja oxigenación del músculo cardíaco que no consigue corresponder a la demanda durante una actividad que exija más esfuerzo.

Seguido a ese problema observamos la presencia del problema Estreñimiento en mitad de la muestra (10). Es definida como una condición en la que la materia fecal en el intestino es muy dura para pasar con facilidad por el trato intestinal, o un estado en que los movimientos de intestino son poco frecuentes y que síntomas incómodos ocu- 
rren. Para la teórica, está clínicamente definido como menos de tres movimientos de intestino por semana (Roy \& Andrews, 1999). El estímulo focal identificado para el estreñimiento fue el reposo en el lecho normalmente prescrito a estos pacientes, disminuyendo así su actividad y movilidad lo que dificulta el peristaltismo del tubo digestivo.

Algunos pacientes relataron hacer uso constante de laxativos mismo en casa para conseguir evacuar con eficiencia. Los pacientes describieron dolor a la evacuación, volumen de heces disminuido, esfuerzo para evacuar, frecuencia disminuida, heces duras y secas. Una dieta rica en fibras y líquidos puede ayudar a estimular la motilidad gastrointestinal. Cuando el estreñimiento se desarrolla, las medidas terapéuticas podrían incluir emolientes fecales, laxativos, supositorios y enemas (Smeltzer \& Bare, 2002).

Los problemas comunes de adaptación deficiencia del sentido primario: visión (6) y audición (6) no son directamente definidos por Roy, mientras la autora afirma que los mismos son congruentes con el diagnóstico de enfermería Trastorno de la percepción sensorial: auditiva y visual, presentes en la taxonomía de la NANDA (Roy \& Andrews, 1999; NANDA, 2005). El estímulo focal para estos problemas fue identificado como la edad, es decir, no hubo influencia directa de la angina en el establecimiento de éstos dos problemas de adaptación.

El tercero más frecuente problema encontrado fue la Nutrición mayor que las necesidades requeridas (07). Este problema es definido como el estado en el cual la persona presenta o está en riesgo de presentar un consumo de nutrientes que exceden las necesidades metabólicas (Roy \& Andrews, 1999). Estos pacientes tiene como ingestión típica una cantidad excesiva de carbohidratos, gorduras, sal, café, refrescos, mismo ya habiendo sido anteriormente prescrita dieta hiposódica, hipocalórica y la eliminación del consumo de café y de refrescos.

Otro problema común identificado fue Deprivación de sueño, observado en 6 personas de la muestra. Este problema de adaptación no está directamente definido por Roy, mientras, la teórica sugiere que el mismo tiene definición equivalente al diagnóstico de mismo nombre presentado en la taxonomía de la NANDA. Este diagnóstico es definido como períodos prolongados de tiempo sin sueño (suspensión natural y periódica del estado de conciencia relativa) (NANDA, 2005). Asociados a esto fueron referidos somnolencia durante el día, cansancio, letargo. Algunos pacientes hacen uso de medicación soporífera para lograr reposar durante la noche por cuenta del ambiente hospitalario, extraño e impersonal que actúa como estímulo focal para este tipo de alteración.

Semejante a éste, encontramos Riesgo de deterioro del patrón de sueño (5), identificado por varios despertares durante la noche, dificultad para dormir y sueño superficial. Este problema de adaptación es definido por Roy como estar en riesgo de presentar alteraciones en los procesos de relajamiento y renovación de energía para actividades futuras (Roy \& Andrews, 1999). El estímulo verificado para esta alteración es la tensión debido su estado de salud caracterizado por miedo, ansiedad, añoranza del lar y de la familia.

Verificamos el problema Hiponatremia (5), definido como niveles séricos de sodio inferiores a $136 \mathrm{mEq} / 1$ lo cual produce mayor excreción de agua por la orina (Roy \& Andrews, 1999). Las principales causas de esta alteración son: terapia diurética prolongada, quemaduras excesivas, sudoresis excesiva, vómitos prolongados, diarrea y nefropatía, ingestión compulsiva de agua, anorexia, alcoholismo, insuficiencia adrenal (Guyton \& Hall, 1998). El estímulo focal identificado fue la prolongada terapia diurética para control de la presión arterial en estos pacientes.

Identificamos también el problema de adaptación denominado Síndrome del desuso (5) del análisis de la habilidad del sujeto en ejecutar su propia alimentación, higiene corporal, higiene íntima, movilidad en el lecho, vestirse, arreglarse y en la movilidad general. Este problema es definido por Roy como Efectos negativos potenciales de actividad física disminuida, particularmente cuando impuestas por restricciones médicas (Roy \& Andrews, 1999). Segundo la orientación de la teórica, este problema incluye todos los tipos de déficit de autocuidado. Constatamos que todos los pacientes poseían habilidad en por lo menos una de las funciones, siendo que los que tenían más necesidad de auxilio, hacían uso de asistencia o supervisión. Apenas uno hacía uso de equipo de auxilio en la Deambulación, debido a amputación. 


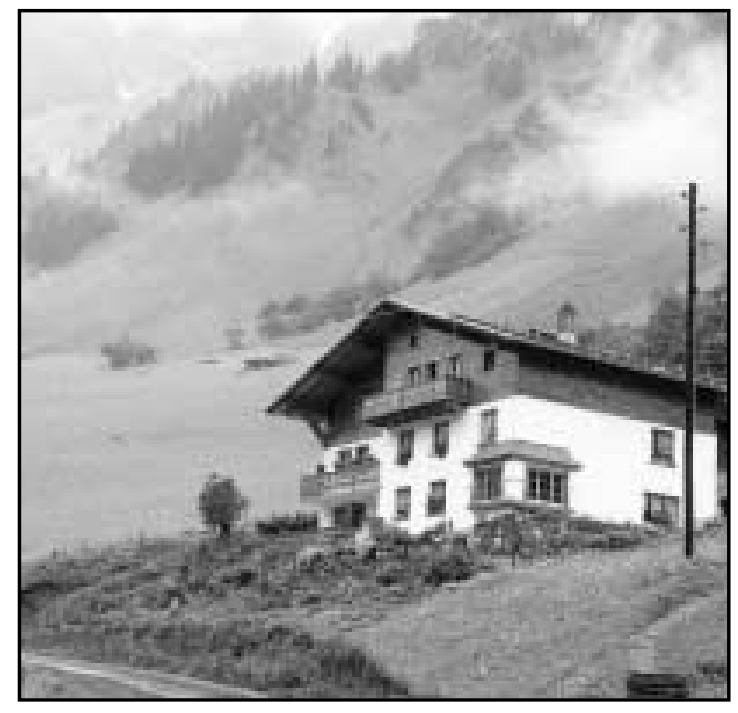

Movilidad restrita (5) fue observada en algunos pacientes que, por motivos específicos tenían problemas de locomoción. Roy define este problema común de adaptación como reducción en los procesos básicos de vida para actividad por medio de los cuales una persona se mueve o es movida (Roy \& Andrews, 1999). Entre los hallazgos había amputación de los dedos de los dos pies debido a diabetes, fractura expuesta en miembro inferior, hematoma extenso y dolorido en miembro inferior y edad avanzada con andar claudicante característico.

La Anorexia (4) significa pérdida o disminución acentuada del apetito provocada por afecciones de origen digestivo, metabólico, endocrino o psíquico. Esta alteración tuvo como estímulo focal los cambios entre los condimentos utilizados en casa y los del hospital, llevándose en cuenta que hay pacientes que no hacen la dieta hiposódica e hipocalórica prescritas. Para mejorar el apetito del paciente, el enfermero debe identificar e incluir las preferencias alimentares del paciente, cuando adecuadas, en la dieta terapéutica prescrita (Roy \& Andrews, 1999; Smeltzer \& Bare, 2002).

El déficit de memoria (4) fue relatado por los propios pacientes como lapsos de memoria reciente. La definición de este problema de adaptación se basa en la disminución de la habilidad para procesar una experiencia por almacenamiento y recuperación de la información (Roy \& Andrews, 1999). Esta alteración es común y está íntimamente rela- cionada al avanzar de la edad y tiene como estímulo focal las alteraciones en los niveles de la presión arterial y en la estructura del endotelio vascular comprometiendo la circulación cerebral.

Con una baja frecuencia, identificamos el problema de adaptación Riesgo de enfrentamiento ineficaz con reacción alérgica (4). Este problema es definido por Roy como un tipo de respuesta inmune en grado exagerado que reconoce moléculas extrañas al organismo y actúa para inactivalas o destruirlas ocasionando reacciones extremas del organismo (Roy \& Andrews, 1999).

Estrés (4) fue también verificado en algunas personas de la muestra, siendo evidente, ya que vivimos en una a sociedad moderna y dinámica. Para Roy, estrés se refiere a la transacción entre las demandas ambientales requeridas para adaptación y los procesos de enfrentamiento reguladores y cognoscitivos individuales (Roy \& Andrews, 1999).

A causa de la globalización de la sociedad, con la consecuente competitividad y estilos de vida modernos y acelerados, los brasileños están menos protegidos de los factores de riesgo para la salud, asociados a enfermedades cardiovasculares. En los últimos años, evidencias han sido acumuladas acerca de la influencia que el estrés tiene sobre el desarrollo de disturbios coronarianos y estudios recientes han demostrado una asociación entre estrés y la manifestación de estas enfermedades. Esto se da a través de la interrelación entre estrés y manifestaciones de orden físico y emocional. Estrés relacionado a un estilo de vida agitado, posiblemente está surgiendo como lo más nuevo y mayor riesgo para el desarrollo de enfermedades cardiovasculares (Franco, Barros, NogueiraMartins \& Michel, 2003).

El problema Edema (3) es caracterizado como la excesiva retención de agua intercelular y la acumulación de líquidos en el espacio intersticial (Roy \& Andrews, 1999). El edema puede resultar de anormalidades vasculares y condiciones sistémicas. En algunos pacientes tuvo asociación con déficit del retorno venoso o alteraciones hidroelectrolíticas.

La Hipocalemia (3) es descrita por niveles séricos de potasio inferiores a $3,5 \mathrm{mEq} / \mathrm{l}$. Puede tener como causas: terapia diurética prolongada, vómi- 
tos prolongados, diarrea, sudoresis intensa, defectos tubulares renales, anorexia, alcoholismo agudo, entre otras. El potasio influencia las actividades de los músculos esqueléticos y cardíacos. Su alteración puede provocar cambios en la irritabilidad y en el ritmo del miocardio. En el caso de los pacientes de este estudio el estímulo focal fue la poliuria, normalmente causada por el uso de diuréticos en el tratamiento de la hipertensión. Pacientes incapacitados o maldispuestos, por un largo período, en ingerir una dieta normal, se encuentran en riesgo de Hipocalemia, ocurriendo con más frecuencia en las personas de edad debilitadas o con anorexia nerviosa. Como prevención, la ingestión de alimentos ricos en potasio debe ser encorajada a través de la educación en salud (Roy \& Andrews, 1999; Smeltzer \& Bare, 2002).

En cuanto a los problemas comunes de adaptación: Infección (1), Instabilidad de comportamiento y humor (1), y Cicatrización comprometida (1), no encontramos asociación plausible con la angina. Afectó cada uno solamente un paciente siendo consideradas alteraciones de carácter individual con calidades particulares a cada paciente. La alteración infección estaba relacionada a un caso de neumonía por Staphilococus aureus, presentando secreción purulenta y fiebre. El caso de comportamiento inestable, la paciente refería nerviosismo, agresividad y hacía uso de benzodiazepínico para control de esas actitudes. Finalmente, con referencia con la cicatrización comprometida, verificamos una úlcera en el miembro inferior izquierdo de un paciente con dificultad de cicatrización por cuenta de diabetes. Estos problemas de adaptación no son directamente definidos por Roy en su libro, mientras, apunta para la posibilidad de utilizarse las definiciones de los diagnósticos enfermeros que componen la taxonomía de la NANDA: Riesgo de infección, afrontamiento defensivo y protección inefectiva.

El problema de adaptación Dolor agudo, común entre los pacientes con angina y relatado en otros estudios (Lopes \& Araujo, 1998; Lopes \& Araujo, 1999) no fue verificado en esta investigación, probablemente porque los pacientes examinados estaban internados hacía más de una semana, bajo efecto de las medicaciones prescritas para alivio de los síntomas de la angina.

\section{BIBLIOGRAFÍA}

- CHRISTENSEN, P.J. \& KENNEY, J.W. (1995). Nursing Process: Application of Conceptual Models. Mosby, Missouri.

- FRANCO G.P., BARROS A.L.B.L., NOGUEIRAMARTINS L.A. \& MICHEL J.L.M. (2003). Stress influence on genesis, onset and maintenance of cardiovascular diseases: literature review. Journal of Advanced Nursing 43: 548-554.

- GUYTON A.C. \& HALL J.E. (2004). Tratado de fisiologia médica. Guanabara Koogan, Rio de Janeiro.

- LOPES, M.V.O. \& ARAÚJO, T.L. (1998). Diagnóstico de enfermagem de ordem física em mulheres com angina pectoris. Rev RENE 1:14-22

- LOPES, M.V.O. \& ARAÚJO, T.L. (1999). Problemas comuns de adaptação fisiológica em mulheres com angina pectoris. Cogitare Enfermagem 4:7-14.

- NANDA (2005). Diagnósticos de enfermagem da NANDA. Artmed, Porto Alegre.

- ROBBINS, S.L., COTRAN, R.S. \& KUMAR, V. (2003). Fundamentos de patologia estrutural e funcional. Guanabara Koogan, Rio de Janeiro.

- ROY, C. \& ANDREWS, H.A. (1999). The Roy Adaptation Model. Appleton \& Lange, Stamford.

- SMELTZER, S.C. \& BARE, B.G. (2002). Tratado de enfermagem médico-cirúrgica. Guanabara Koogan, Rio de Janeiro.

- SOCIEDADE BRASILEIRA DE CARDIOLOGIA (2004). Diretrizes de doença coronariana crônica angina estável. Arquivos Brasileiros de Cardiologia. 83: suplemento 2 . 